\title{
Etude de la relation phylogénétique entre trois morphotypes de Solenostemon rotundifolius (Poir J. K. Morton) originaires du Burkina Faso par les marqueurs microsatellites chloroplastiques (SSRcp)
}

\author{
Romaric Kiswendsida NANEMA ${ }^{1 *}$, Antoine Abel MISSIHOUN ${ }^{2}$, Clément AGBANGLA ${ }^{2}$, \\ Corneille AHANHANZO ${ }^{2}$, Ernest Renan TRAORE ${ }^{1}$, Pauline BATIONO/KANDO ${ }^{1}$, \\ Mahamadou SAWADOGO ${ }^{1}$ and Jean-Didier ZONGO $^{1}$ \\ ${ }^{1}$ Laboratoire de Génétique et de Biotechnologie Végétales, Unité de Formation et de Recherche en Sciences \\ de la Vie et de la Terre, Université de Ouagadougou 03, BP 7021 Ouagadougou 03, Burkina Faso. \\ ${ }^{2}$ Laboratoire de Génétique et des Biotechnologies, Faculté des Sciences et Techniques, Université d'Abomey- \\ Calavi, Benin. \\ *Auteur correspondant ; E-mail: romaric_nanema@univ-ouaga.bf ; nanemaromaric@yahoo.fr; \\ Tél: 0022678846765
}

\section{RESUME}

Solenostemon rotundifolius est une plante à tubercules cultivée en Afrique de l'Ouest et particulièrement sur le plateau central du Burkina Faso. Mais comme la plupart des Lamiacées, sa biologie est encore peu connue. Les travaux de caractérisation de la variabilité morphologique ont révélé la présence de trois morphotypes sur la base de la couleur de la peau des tubercules mais les différences morphologiques pourraient faire penser à des espèces différentes. L'objectif de la présente étude a été d'étudier la relation phylogénétique entre ces morphotypes par les marqueurs microsatellites chloroplastiques (SSRcp) développés sur les Solanacées. Au total, 10 amorces ont été testées sur 12 accessions originaires du Burkina Faso. Sur l'ensemble de ces amorces, 5 ont réussi à amplifier l'ADN chloroplastique de $S$. rotundifolius. Les résultats ont montré un monomorphisme pour tous les 5 marqueurs traduisant la proximité phylogénétique des trois morphotypes. Ce qui confirmerait la même appartenance spécifique de ces trois morphotypes.

() 2010 International Formulae Group. All rights reserved.

Mots clés : Tubercules, morphotypes, SSRcp, phylogénétique.

\section{INTRODUCTION}

Solenostemon rotundifolius appartient à la famille des Lamiacées (Labiées) qui compte 3000 à 3500 espèces d'herbes, d'arbres et d'arbrisseaux très cosmopolites. Les Lamiacées sont phylogénétiquement plus proches des Verbenacées que des autres familles botaniques voisines (Wagstaff et Olmstead, 1997). La plasticité écologique, les vertus alimentaires et médicinales des
Lamiacées ont suscité récemment un intérêt particulier pour leurs ressources génétiques (Odalo et al., 2005; Owuor et Kisangau, 2005 ; Busmann et al., 2006 ; Lakušić et al., 2006 ; Lisboa et al., 2006 ; Uniyal et al., 2006 ; Gulcin et al., 2007).

$S$. rotundifolius est une herbacée annuelle des zones tropicales (Ryding, 1994). Elle serait originaire d'Afrique de l'Est ou du Centre (Schoeninger et al., 2000 ; Abraham et 
Radhakrishnan, 2005 ; Edison et al., 2006). En Afrique de l'Ouest, elle a été largement cultivée pour ses tubercules comestibles (Chevalier et Perrot, 1905). Au Burkina Faso, sa culture s'est principalement développée autour du plateau central entre 700 et $800 \mathrm{~mm}$ d'eau par an (Ouédraogo et al., 2007). Ses potentialités alimentaires et sa bonne adaptation aux conditions pédoclimatiques du Sahel ont suscité des travaux de caractérisation de son système de culture (Ouédraogo et al., 2007) et sur la variabilité agromorphologique de ses ressources génétiques (Nanéma et al., 2009). Mais, comme la plupart des Lamiacées, la biologie de $S$. rotundifolius n'est pas bien connue et de nombreuses ambiguïtés existent encore dans la description de ses ressources génétiques (Trusty et al., 2004).

La caractérisation de la variabilité agromorphologique des ressources génétiques de $S$. rotundifolius du Burkina Faso a révélé l'existence de trois couleurs de la peau du tubercule (Nanéma et al., 2009). Il s'agit des colorations noirâtre, rougeâtre et blancjaunâtre. Ces colorations de tubercules ont été des critères de base pour la description variétale ou morphotypique au sein de l'espèce (Chevalier et Perrot, 1905; NRI, 1987 ; Jayakody et al., 2005 ; Prematilake, 2005 ; Tanzubil et al., 2005). Cependant, l'identification de formes florales différentes au sein du germplasme de S. rotundifolius (Opoku-Agyema et al., 2007 ; Nanéma et al., 2009) nous ont conduit à l'hypothèse de l'existence d'espèces différentes cultivées sous l'appellation $S$. rotundifolius. La description botanique faite des variétés par Chevalier et Perrot (1905) reste incomplète et est à l'origine de quelques ambiguïtés.

Depuis la découverte de l'ADN cytoplasmique, des travaux ont été menés sur sa structure (Charret et André, 1968). L'hérédité monoparentale de l'ADN cytoplasmique, a permis de mettre au point des techniques d'études phylogénétiques chez les plantes. Actuellement, le développement des microsatellites chloroplastiques (SSRcp) a permis de mettre au point des marqueurs afin de permettre l'analyse du polymorphisme de l'ADN chloroplastique chez plusieurs espèces (Bryan et al., 1999; Weising et Gardner, 1999). Ces marqueurs ont permis l'analyse des relations phylogénétiques au niveau interspécifique (Grivet et al., 2003 ; De Mattia et al., 2008 ; Basha et Sujatha, 2009) mais aussi au niveau intra spécifique (AdamBlondon et al., 2005 ; Grassi, 2006 ; Imazio et al., 2006 ; Wills et Burke, 2006 ; Boccacci et Betta, 2009 ; Pastorino et al., 2009).

Les marqueurs microsatellites sont devenus un puissant outil dans l'analyse des relations phylogénétiques du fait de leur applicabilité à plusieurs espèces souvent peu connues comme $S$. rotundifolius. Le but de la présente étude est d'étudier la relation phylogénétique entre les morphotypes identifiés au sein du germplasme de $S$. rotundifolius. Il s'agit du morphotype (A) à tubercules à peau rougeâtre, du morphotype (B) à tubercules à peau noirâtre et du morphotype $(\mathrm{C})$ à tubercules à peau blancjaunâtre (Nanéma et al., 2009).

\section{MATERIEL ET METHODES \\ Matériel végétal \\ Origine et caractéristiques du matériel végétal}

L'étude de la phylogénie a porté sur des accessions de $S$. rotundifolius issues d'une collection du germplasme de $S$. rotundifolius réalisée en 2007 au Burkina Faso par le Laboratoire de Génétique et de Biotechnologie Végétale de l'Université de Ouagadougou (Burkina Faso) (Nanéma et al., 2009). Ces accessions ont fait l'objet d'une caractérisation agromorphologique en 2007. $\mathrm{Au}$ total, 12 accessions ayant montré une homogénéité morphologique lors de l'évaluation agromorphologique ont été retenues.

Le nombre d'accessions par morphotype a été défini sur la base de la fréquence des trois morphotypes dans la collection. La localité d'origine des accessions a été aléatoirement choisie. Ainsi, sur les 12 accessions retenues, 8 ont été à tubercules à peau rougeâtre (morphotype A), 3 ont été du 
morphotype à tubercules à peau noirâtre (B) et une accession a été à tubercules à peau blancjaunâtre (C). La Figure 1 montre la répartition géographique des accessions. Les accessions à tubercules à peau noirâtre proviennent des provinces du Bazèga, du Noumbiel et du Nahouri. Celles à tubercules à peau rougeâtre proviennent des provinces du Bazèga, du Nahouri, du Noumbiel, du Sanguié, du Sanematenga et du Zoundwéogo. L'accession à tubercules à pelure blanc-jaunâtre provient de la province du Nahouri.

\section{Mise en culture des semenceaux}

Pour chaque accession retenue, 10 tubercules ont été aléatoirement tirés. Ces tubercules avaient déjà germé en stockage. La mise en culture des semenceaux a été faite dans la serre du Laboratoire de Génétique et des Biotechnologies de la Faculté des Sciences et Techniques (FAST) de l'Université d'Abomey-Calavi (République du Bénin). Dix pots de culture remplis de terreau ont été préparés pour chaque accession le 17 Mars 2009 suivi d'un arrosage abondant. Le repiquage des semenceaux dans les pots a été fait le 18 Mars 2009. Les tubercules ont été individuellement mis en terre dans chaque pot de sorte que le bourgeon apical affleure légèrement. Le dispositif ainsi mis en place a été quotidiennement arrosé en évitant le dessèchement et l'engorgement. La croissance des plantules a été suivie jusqu'à la formation des premières feuilles.

\section{Prélèvement du matériel végétal}

Le matériel végétal a été prélevé dix jours après le repiquage des semenceaux. Pour chaque accession, quatre pieds aléatoirement retenus ont été consécutivement numérotés, soit au total 48 pieds pour l'ensemble des accessions. 0,2 $\mathrm{g}$ de feuilles fraîches ont été prélevés sur chaque pied. Pour certains pieds pour lesquels la levée n'a pas été effective, 0,8 $\mathrm{g}$ du tubercule repiqué ont été prélevés. Le matériel végétal ainsi récolté et numéroté a été conservé au réfrigérateur $\left(4{ }^{\circ} \mathrm{C}\right)$ avant l'extraction d'ADN.

\section{Extraction et dosage de l'ADN}

La méthode d'extraction de l'ADN est celle mise au point par Agbangla et al. (2002) avec quelques modifications. Chaque échantillon, feuilles ou tubercule, a été finement broyé dans $2 \mathrm{ml}$ de solution tampon Tris-EDTA-Sorbitol (TES) à l'aide d'un mortier. Le broyat de chaque échantillon recueilli dans un tube eppendorf a été centrifugé à 10000 RPM (rotations par minute) pendant $10 \mathrm{mn}$ à $4{ }^{\circ} \mathrm{C}$ avec le frein à 4. Le surnageant a été éliminé et $750 \mu \mathrm{l}$ de tampon MATAB (Mixed Alkyl Trimethyl Amonium Bromide) préchauffé à $65^{\circ} \mathrm{C}$ a été ajouté au culot. Les tubes ont été agités afin de reprendre complètement le culot dans le tampon de lyse. Ils ont ensuite été incubés à $65^{\circ} \mathrm{C}$ dans un bain-marie pendant 2 heures 30 minutes. Le contenu des tubes a été homogénéisé par retournement chaque $15 \mathrm{mn}$.

A la fin de l'incubation, les tubes ont été refroidis pendant quelques minutes à la température ambiante de la salle de manipulation. $750 \mu \mathrm{l}$ du mélange chloroforme et alcool isoamylique (CIAA) dans les proportions 24:1 a été ajouté à chaque tube. Les tubes ont par la suite été centrifugés à 10000 RPM pendant $15 \mathrm{mn}$ à $4{ }^{\circ} \mathrm{C}$ sans freinage. Le surnageant a été récupéré dans un nouveau tube. De l'isopropanol très frais $(-20$ ${ }^{\circ} \mathrm{C}$ ) à volume égal du surnageant a été ajouté dans chaque tube afin de précipiter l'ADN. Les tubes ont été agités par retournement jusqu'à apparition de la pelote d'ADN. Ils ont été à nouveau centrifugés à 10000 RPM à 4 ${ }^{\circ} \mathrm{C}$ pendant $10 \mathrm{mn}$ avec le frein à 4 . Le surnageant a été éliminé et les tubes ont été mis à sécher à température ambiante. La pelote d'ADN a été reprise dans un tampon TE (Tris-EDTA 1X). Les tubes ont été conservés dans un congélateur à $-20{ }^{\circ} \mathrm{C}$. La concentration et la qualité de l'ADN dans chaque tube ont été vérifiées à l'aide d'un spectrophotomètre BIO RAD, Smart Spec Plus.

Caractéristiques des amorces microsatellites chloroplastiques testées

Dix amorces microsatellites chloroplastiques SSRcp ont été testées. Il s'agit de 9 amorces Ntcp (Nicotiana tabacum chloroplast) développées sur le tabac (Bryan et al., 1999) et d'une amorce ccmp (consensus 
chloroplast microsatellite primers), amorces universelles développées par Weising et Gardner (1999). Il s'agit des amorces Ntcp 5, Ntcp 8, Ntcp 9, Ntcp 15, Ntcp 25, Ntcp 26, Ntcp 30, Ntcp 34, Ntcp 36 et de ccmp 2 (Tableau 1). Les températures d'hybridation varient entre 50 et $60^{\circ} \mathrm{C}$. La taille des locus obtenus chez le tabac (Nicotiana tabacum) varie entre 120 et $251 \mathrm{pb}$ (paires de bases) respectivement pour Ntcp 36 et Ntcp 8.

\section{Amplification PCR}

Pour chaque échantillon, un volume de $25 \mu \mathrm{l}$ de produit PCR contenant $25 \mathrm{ng}$ d'ADN génomique, $0,2 \mu \mathrm{M}$ de chaque amorce, 150 $\mu \mathrm{M}$ de dNTPs, $2,5 \mathrm{mM}$ de $\mathrm{MgCl}_{2}, 1 \mathrm{X}$ de tampon PCR et $1 \mathrm{U}$ de Tap ADN polymerase a été préparé dans un tube eppendorf. Après homogénéisation, les tubes ont été placés dans un thermocycleur de type Peltier-Effect Cycling PTC 100 programmé pour une phase initiale de dénaturation de l'ADN à $94{ }^{\circ} \mathrm{C}$ pendant $4 \mathrm{mn}$, suivie de 36 cycles de dénaturation à $94{ }^{\circ} \mathrm{C}$ pendant $30 \mathrm{~s}$, d'hybridation à $55{ }^{\circ} \mathrm{C}$ pendant $1 \mathrm{mn}$, de synthèse à $72{ }^{\circ} \mathrm{C}$ pendant $1 \mathrm{mn}$ et d'une phase de synthèse finale à $72{ }^{\circ} \mathrm{C}$ pendant $5 \mathrm{mn}$. Après ajout de $25 \mu \mathrm{l}$ de bleu stop (2X) dans chaque tube, les produits PCR ont été dénaturés à $92^{\circ} \mathrm{C}$ pendant $3 \mathrm{mn}$ et conservés à $72{ }^{\circ} \mathrm{C}$ jusqu'au dépôt sur gel pour la migration électrophorétique.

\section{Electrophorèse et lecture des bandes}

La migration électrophorétique a été faite sur un gel dénaturant d'acrylamide à 5\% de dimensions $305 \times 385 \mathrm{~mm}$. Après le montage du gel dans une cuve à électrophorèse verticale, les chambres inférieure et supérieure de la cuve ont été remplies avec du tampon TBE (Tris-BorateEDTA) $1 \mathrm{X}$. Un préchauffage du gel pendant 1 $\mathrm{h}$ à $60 \mathrm{~W}$ a été fait. Un volume de $10 \mu \mathrm{ldu}$ mélange (produit PCR et bleu stop) a été déposé dans chaque puit. La migration des produits PCR a été faite pendant $1 \mathrm{~h} 30 \mathrm{mn}$ à 2 h. La révélation des bandes a été faite au nitrate d'argent selon la méthode de Creste et al. (2001).

Les plaques électrophorétiques ont été lues à la lumière blanche d'un transilluminateur Waldmann W. Les bandes ont été identifiées sur la base de leur position sur le gel. Ainsi la présence (1) et l'absence
(0) des bandes ont été enregistrées pour chaque individu et pour l'ensemble des amorces testées.

\section{RESULTATS \\ Quantité et qualité de l'ADN extrait}

La technique d'extraction appliquée a permis d'extraire l'ADN total à partir des jeunes feuilles mais aussi des tubercules de $S$. rotundifolius. Le dosage au spectrophotomètre a donné en moyenne une concentration en

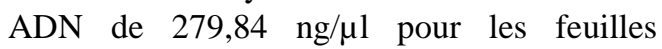
fraîches et de $255 \mathrm{ng} / \mu \mathrm{l}$ pour les tubercules dans un volume total de $150 \mu \mathrm{l}$ de tampon. Le rapport A260/A280 (absorbance à 260 $\mathrm{nm} /$ absorbance à $280 \mathrm{~nm}$ ) a varié en moyenne entre 1 et 2 aussi bien pour l'ADN extrait à partir des feuilles que pour celui extrait à partir des tubercules.

Les tests préliminaires d'amplification PCR ont montré que les quantités d'ADN comprises entre $15 \mathrm{ng}$ et $30 \mathrm{ng}$ par tube selon les conditions d'amplifications employées, étaient les quantités optimales pour l'amplification. Ce qui correspond à 0,6 à 1,2 $\mathrm{ng} / \mu \mathrm{l}$ du mélange PCR préparé. Ces résultats ont conduit à fixer la quantité d'ADN par tube à $25 \mathrm{ng}$ pour la suite des travaux.

\section{Locus microsatellites observés}

Sur les dix amorces microsatellites chloroplastiques testées, cinq n'ont donné aucune bande sur l'ensemble des individus de toutes les accessions. Il s'agit de Ntcp 5, Ntcp 15, Ntcp 25, Ntcp 30 et de Ntcp 36. Sur le génome chloroplastique de $N$. tabacum ces amorces amplifient des locus microsatellites à T14 (cas de Ntcp 5 et de Ntcp 30), à A10 (Ntcp 15), à A13 (Ntcp 9) et à T13 et T15 (Ntcp 30) avec des bandes dont la taille varie entre 120 et $190 \mathrm{pb}$.

Les cinq autres amorces, Ntcp 8, Ntcp 9, Ntcp 26, Ntcp 34 et ccmp 2 ont donné des locus monomorphes pour l'ensemble des individus des 12 accessions. Sur l'ADN chloroplastique de N. tabacum, ces amorces amplifient des régions microsatellites à T10 (Ntcp 9, Ntcp 26 et Ntcp 34), à T11 (Ntcp 8) et à A11 (ccmp 2).

Répartition haplotypique des morphotypes de $S$. rotundifolius 
Les individus caractérisés appartiennent à trois morphotypes distincts identifiés chez $S$. rotundifolius. Il s'agit du morphotype à tubercules à peau de couleur rougeâtre $(\mathrm{A})$, de celui à tubercules à peau de couleur noirâtre (B), et du morphotype à tubercules à peau de couleur blanc-jaunâtre (C). Les morphotypes ont montré une homogénéité pour toutes les amorces testées (Photo 1). Pour les cinq locus observés, les morphotypes ont révélé des haplotypes identiques.

\section{DISCUSSION}

Les amorces microsatellites chloroplastiques testées sur les trois morphotypes de $S$. rotundifolius (Ntcp et ccmp) ont été développées sur les Solanacées avec pour objectif de permettre leur application dans l'étude de la variabilité de l'ADN chloroplastique de plusieurs autres espèces ou familles d'Angiospermes (Bryan et al., 1999 ; Weising et Gardner, 1999). Comme dans le cas de la présente étude, l'absence d'amplification avec certaines amorces a été aussi observée avec plusieurs espèces telles que Vitis vinifera, Helianthus annuus, Dioscorea sp., et des espèces de la famille des Dipterocarpacées (Chaïr et al., 2005 ; Will et al., 2005; Arroyo-Garcia et al., 2006 ; Indrioko et al., 2006). Selon Decroocq et al. (2004), l'absence d'amplification s'explique par l'absence de la région correspondante ciblée par l'amorce sur l'ADN chloroplastique. L'absence d'amplification chez les trois morphotypes de $S$. rotundifolius identifiés serait due à l'absence chez ces morphotypes de la région microsatellite ciblée par les amorces. Cinq sur dix, soit 50\% des amorces développées sur les Solanacées n’ont pas réussi à amplifier une région spécifique sur l'ADNcp chez les trois morphotypes de $S$. rotundifolius. Ces différentes observations montrent la limite de l'universalité de ces amorces telles que visées, mais surtout le besoin de développer pour chaque famille de plante, des amorces spécifiques.

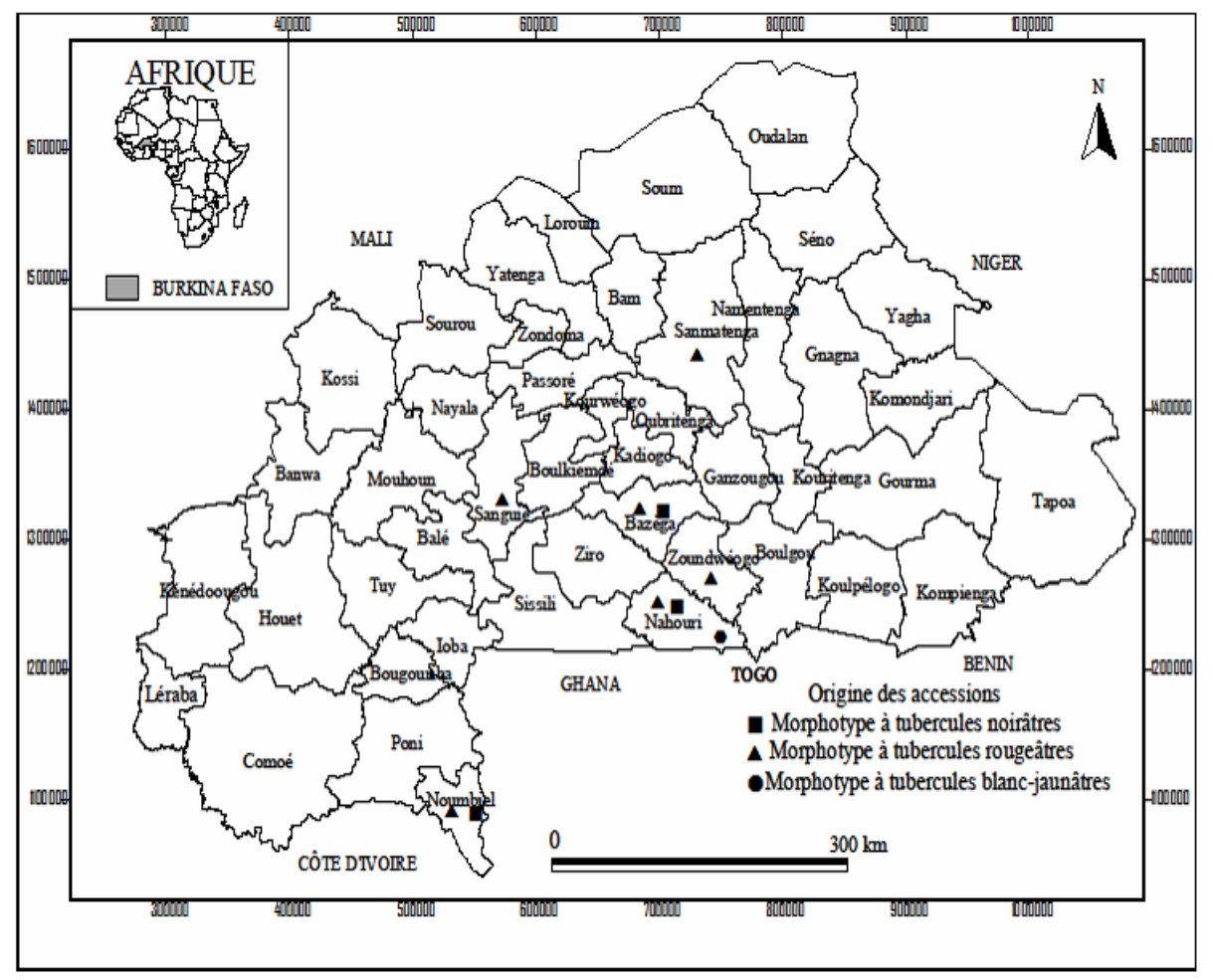

Figure 1 : Origine des accessions retenues pour l'étude des relations phylogénétiques entre les trois morphotypes de $S$. rotundifolius. 


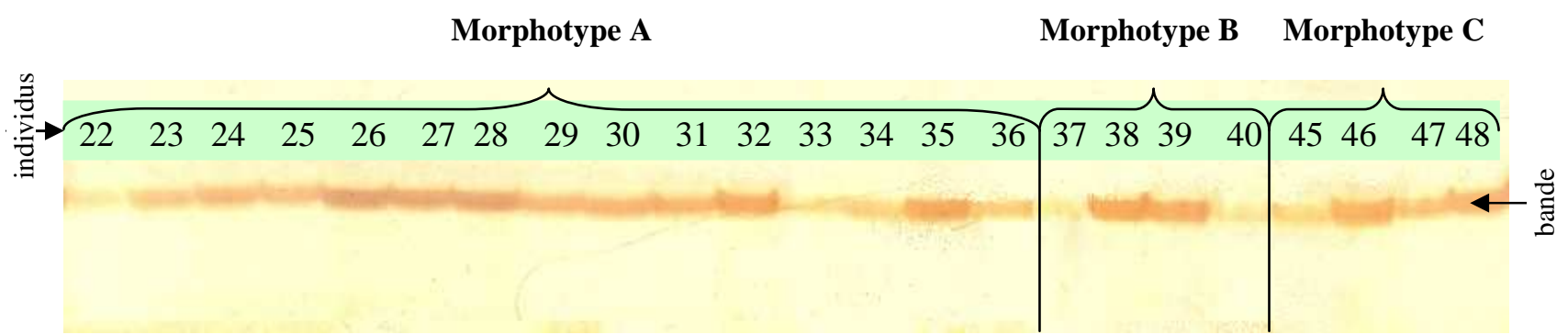

Photo 1 : Electro-phorégramme des trois morphotypes de $S$. rotundifolius (amorce Ntcp 8) montrant

leur monomorphisme et leur homogénéité.

Morphotype $\mathrm{A}$ : Morphotype à tubercules à peau rougeâtre ;

Morphotype B : Morphotype à tubercules à peau noirâtre

Morphotype $\mathrm{C}$ : Morphotype à tubercules à peau blanc-jaunâtre. 
Tableau 1 : Caractéristiques des amorces microsatellites testées dans l'analyse phylogénétique des morphotypes de $S$. rotundifolius.

\begin{tabular}{|c|c|c|c|c|c|}
\hline Code & Localisation & Répétition & Amorce (5'- 3') & $\mathbf{T}\left({ }^{\circ} \mathbf{C}\right)$ & $\begin{array}{l}\text { Taille chez } \\
N . \\
\text { tabacum } \\
\text { (pb) }\end{array}$ \\
\hline Ntcp5 & $\begin{array}{l}\text { rps16/trnQ } \\
\text { région } \\
\text { intergénique }\end{array}$ & T14 & $\begin{array}{l}\text { CGAATTGATAGATACGAAACC } \\
\text { AATACACCAAACAACAAATCC }\end{array}$ & 54 & 150 \\
\hline Ntcp8 & $\begin{array}{l}\text { trnG } \\
\text { intron }\end{array}$ & $\mathrm{T} 11$ & $\begin{array}{l}\text { ATATTGTTTTAGCTCGGTGG } \\
\text { TCATTCGGCTCCTTTATG }\end{array}$ & 55 & 251 \\
\hline Ntcp9 & $\begin{array}{l}\text { trnG/trnR } \\
\text { région } \\
\text { intergénique }\end{array}$ & $\mathrm{T} 10$ & $\begin{array}{l}\text { CTTCCAAGCTAACGATGC } \\
\text { CTGTCCTATCCATTAGACAATG }\end{array}$ & 55 & 237 \\
\hline Ntcp15 & & $\mathrm{A} 10$ & $\begin{array}{l}\text { TGCAAAAATCCTACTCTTC } \\
\text { TCTTTCGATTATTTTAGTTTAGA }\end{array}$ & 50 & 120 \\
\hline Ntcp25 & $\begin{array}{l}\text { atpB/rbcL } \\
\text { région } \\
\text { intergénique }\end{array}$ & A13 & $\begin{array}{l}\text { TTAGTCAGGTATTTCCATTTC } \\
\text { CTTTTCATAGGAATCTTTCACA }\end{array}$ & 50 & 190 \\
\hline Ntcp26 & $\begin{array}{l}\text { psal/ORF184 } \\
\text { région } \\
\text { intergénique }\end{array}$ & $\mathrm{T} 10$ & $\begin{array}{l}\text { GCAATTGCAATGGCTTCTTTA } \\
\text { TTTATGTTCGGTGGAAATCACA }\end{array}$ & 60 & 168 \\
\hline Ntcp30 & $\begin{array}{l}\text { clpP } \\
\text { intron }\end{array}$ & T13.T15 & $\begin{array}{l}\text { GATGGCTCCGTTGCTTTAT } \\
\text { TGCCGGAGAGTTCTTAACAATA }\end{array}$ & 60 & 158 \\
\hline Ntcp34 & $\begin{array}{l}\text { rps8/rpl14 } \\
\text { région } \\
\text { intergénique }\end{array}$ & $\mathrm{T} 10$ & $\begin{array}{l}\text { GATCCATATCAGCATTTCGTAT } \\
\text { CTGTTTCTAGTGGGGTATTTGA }\end{array}$ & 60 & 212 \\
\hline Ntcp36 & $\begin{array}{l}\text { lps19/rp12 } \\
\text { région } \\
\text { intergénique }\end{array}$ & T14 & $\begin{array}{l}\text { GTAGTAAATAGGAGAGAAAATAGA } \\
\text { TGATACATAGTGCGATACAG }\end{array}$ & 50 & 125 \\
\hline ccmp2 & 5 ' to trnS & A11 & $\begin{array}{l}\text { GATCCCGGACGTAATCCTG } \\
\text { ATCGTACCGAGGGTTCGAAT }\end{array}$ & 60 & 189 \\
\hline
\end{tabular}

Ccmp : consensus chloroplast microsatellite primers, Ntcp : Nicotiana tabacum chloroplast.

La moitié des amorces utilisées a donné des amplifications et a révélé un monomorphisme pour l'ensemble des morphotypes étudiés. L'application de ces amorces Ntcp et ccmp dans d'autres études a permis de révéler une variabilité interspécifique au sein de nombreuses familles et genres de plantes. Ces amorces ont révélé un polymorphisme interspécifique dans les genres Pinus (Powell et al., 1995 ; Gugerli et al., 2001), Quercus (Deguilloux et al., 2004), Vitis (De Mattia et al., 2008), Prunus (Petitpierre et al., 2009), Carpinus (Grivet et al., 2003), Capersicum (Ryzhova et Kochieva, 2004), Jatropha (Basha et Sujatha, 2009), Citrus (Cheng et al., 2005) et au sein de la famille des Dipterocarpacées (Indrioko et al., 2006 ; Rachmayanti et al., 2006). Chez des plantes à tubercules, les amorces testées ont révélé un polymorphisme interspécifique au sein des genres Solanum (Sukhotu et Hosaka, 2006) et Dioscorea (Chaïr et al., 2005).
Les travaux de Fineschi et al. (2002), Indrioko et al. (2006), et de Rachmayanti et al. (2006) ont montré l'homogénéité intraspécifique avec les marqueurs microsatellites chloroplastiques. Certains travaux ont montré l'existence d'une faible variabilité intraspécifique révélée par les marqueurs microsatellites chloroplastiques. C'est le cas chez Helianthus annuus (Wills et Burke, 2006), Hagenia abyssinaca (Ayele et al., 2009), Nothofagus antartica (Pastorino et al., 2009), Vitis vinifera (Adam-Blondon et al., 2005 ; Grassi, 2006 ; Imazio et al., 2006) et chez Corylus avellana (Boccacci et Betta, 2009). Ces marqueurs ont aussi révélé une variabilité intraspécifique chez la pomme de terre (Raimondi et al., 2005 ; Martyrosyan et al., 2007), chez des hybrides somatiques de Citrus sp (Cheng et al., 2003) et chez le riz, le maïs et le blé (Provan et al., 2004). Ces différents travaux soutiennent le fait que le monomorphisme observé pour les cinq locus 
avec les trois morphotypes est en faveur de leur appartenance à une même espèce.

\section{Conclusion}

Les amorces développées sur des Solanacées ont réussi à amplifier des séquences microsatellites de l'ADN chloroplastique de $S$. rotundifolius de la famille des Lamiacées. Pour les cinq locus révélés, les trois morphotypes de $S$. rotundifolius ont montré une homogénéité intra et inter morphotypique. Malgré les différences morphologiques entre les trois morphotypes, les résultats sont en faveur de leur appartenance à une même espèce.

Ce présent travail est l'une des premières initiatives sur la biologie moléculaire de $S$. rotundifolius et il serait intéressant pour la suite de l'étude de tester un plus grand nombre d'amorces microsatellites chloroplastiques tout en intégrant d'autres espèces de la famille des Lamiacées. La collecte d'échantillons dans plusieurs pays de la sous région pourrait permettre de tester la répartition géographique des haplotypes qui seront identifiés. Ceci contribuera à approfondir la connaissance de la phylogénie au sein des Lamiacées et, peut être, à pallier les insuffisances taxonomiques au sein de la famille.

\section{BIBLIOGRAPHIE}

Agbangla C, Ahanhanzo C, Tostain S, Dansi A, Daïnou O. 2002. Evaluation de la diversité génétique par RAPD d'un échantillon de Dioscorea alata d'une région du Bénin, la sous-préfecture de Savè. J. Rech. Sci. Univ. Lomé, 6(1): $197-$ 202.

Abraham M, Radhakrishnan VV. 2005. Assessment and induction of variability in coleus (Solenostemon rotundifolius). Indian Journal of Agricultural Sciences, 75(12): 834-836.

Adam-Blondon A-F, Bernole A, Faes G, Lamoureux D, Pateyron S, Grando MS, Caboche M, Velasco R, Chalhoub B. 2005. Construction and characterization of BAC libraries from major grapevine cultivars. Theor. Appl. Genet., 110: 13631371.

Arroyo-García R, Ruiz-García L, Bolling L, Ocete R, López MA, Arnold C, Ergul A, Söylemezoğlu G, Uzun hI, Cabello f, Ibáñez J, Aradhya MK, Atanassov A, Atanassov I, Balint S, Cenis JL, Costantini L, Gorislavets S, Grando MS, Y Klein B, Mcgovern PE, Merdinoglu D,
Pejic I, PELSY F, Primikirios N, Risovannaya V, Roubelakis-Angelakis KA, Snoussi H, Sotiri P, Tamhankar S, This P, Troshin L, Malpica JM, Lefort F, Martinez-Zapater JM. 2006. Multiple origins of cultivated grapevine (Vitis vinifera L. ssp sativa) based on chloroplast DNA polymorphisms. Molecular Ecology, 15: 3707-3714.

Ayele TB, Gailing O, Umer M, Finkeldey R. 2009. Chloroplast DNA haplotype diversity and postglacial recolonization of Hagenia abyssinica (Bruce) JF Gmel in Ethiopia Plant. Syst. Evol., 280:175-185.

Basha SD, Sujatha M. 2009. Genetic analysis of Jatropha species and interspecific hybrids of Jatropha curcas using nuclear and organelle specific markers. Euphytica, 168: 197-214.

Boccacci P, Botta R. 2009. Investigating the origin of hazelnut (Corylus avellana L.) cultivars using chloroplast microsatellites. Genet. Resour. Crop Evol., 56: 851-859.

Bryan GJ, McNicoll J, Ramsay G, Meyer RC, Jong WSD. 1999. Polymorphic simple sequence repeat markers in chloroplast genomes of Solanaceous plants. Theor. Appl. Genet., 99: 859-867.

Busmann RW, Gilbreath GG, Solio J, Lutura M, Lutuluo R, Kunguru K, Wood N, Mathenge SG. 2006. Plant use of the Maasai of Sekenani Valley, Maasai Mara, Kenya. Journal of Ethnobiology and Ethnomedicine, 2: 22.

Chaïr H, Perrier X, Agbangla C, Marchand JL, Daïnou O, Noyer JL. 2005. Use of cpSSRs for the characterisation of yam phylogeny in Benin. Genome, 48: 674684.

Charret R, André J. 1968. La synthèse de L'ADN mitochondrial chez Tetrahymena pyriformis: Etude radioautographique quantitative au microscope électronique. The Journal of Cell. Biology, 39: 369381.

Cheng Y, De Vicente MC, Meng H, Guo W, Tao N, Deng X. 2005. A set of primers for analyzing chloroplast DNA diversity in Citrus and related genera. Tree Physiology, 25: 661-672.

Cheng Y-J, Guo W-W, Deng X-X. 2003. cpSSR : a new tool to analyse chloroplast genome of Citrus somatic hybrids. Acta Botanica Sinica, 45(8): 906-909.

Chevalier A, Perrot E. 1905. Les Coleus à tubercules alimentaires. In Les Végétaux utiles de l'Afrique Tropicale Française: 
Etudes Scientifiques et Agronomiques, Vol I-Fasc I : Paris ; 100-152.

Creste S, Neto AT, Figueira A. 2001. Detection of single sequence repeat polymorphisms in denaturing polyacrylamide sequencing gels by silver staining. Plant. Mol. Biol. Rep., 19: 299306.

De Mattia F, Imazio S, Grassi F, Baneh HD, Scienza A, Labra M. 2008. Study of genetic relationships between wild and domesticated grapevine distributed from Middle East regions to european countries. Rendiconti Lincei, 19: 223-240.

Decroocq V, Hagen LS, Favé M-G, Eyquard J-P, Pierronnet A. 2004. Microsatellite markers in the hexaploid Prunus domestica species and parentage lineage of three European plum cultivars using nuclear and chloroplast simple-sequence repeats. Molecular Breeding, 13: 135 142.

Deguilloux M, Pemonge M, Petit RJ. 2004. Use of chloroplast microsatellites to differentiate oak populations. Ann. For. Sci., 61: 825-830.

Edison S, Unnikrishnan M, Vimala B, Pillai SV, Sheela MN, Sreekumari MT, Abraham K. 2006. Biodiversity of Tropical Tuber Crops in India national Biodiversity Authority National. Biodiversity Authority Chennai, Tamil Nadu: India.

Fineschi S, Anzidei M, Cafasso D, Cozzolino S, Garf G, Pastorelli R, Salvini D, Taurchini D, Vendramin GG. 2002. Molecular markers reveal a strong genetic differentiation between two European relic tree species: Zelkova abelicea (Lam) Boissier and Z. sicula Di Pasquale, Garfi \& Quézel (Ulmaceae). Conservation Genetics, 3: 145-153.

Grassi F, Labra M, Imazio S, Rubio RO, Failla O, Scienza A, Sala F. 2006. Phylogeographical structure and conservation genetics of wild grapevine. Conservation Genetics, 7: 837-845.

Grivet D, Petit RJ. 2003. Chloroplast DNA phylogeography of the hornbeam in Europe: Evidence for a bottleneck at the outset of postglacial colonization. Conservation Genetics, 4: 47-56.

Gugerli F, Senn J, Anzidei M, Madaghiele A, Büchler U, Sperisen C, Vendramin GG. 2001. Chloroplast microsatellites and mitochondrial nadl intron 2 sequences indicate congruent phylogenetic relationships among Swiss stone pine
(Pinus cembra), Siberian stone pine (Pinus sibirica), and Siberian dwarf pine (Pinus pumila). Molecular Ecology, 10: 1489-1497.

Gulcin I, Elmastas M, Aboul-Enein HY. 2007. Determination of antioxydant and radical scavenging activity of Basil (Ocimum basilicum L. Family Lamiaceae) assayed by different methodologies. Phytother. Res., 21(4): 354-61.

Imazio S, Labra M, Grassi1 F, Scienza A, Failla O. 2006 Chloroplast microsatellites to investigate the origin of grapevine. Genetic Resources and Crop Evolution, 53: 1003-1011.

Indrioko S, Gailing O, Finkeldey R. 2006 Molecular phylogeny of Dipterocarpaceae in Indonesia based on chloroplast DNA. Pl. Syst. Evol., 261: 99115.

Jayakody L, Hoover R, Liu Q, Weber E. 2005. Studies on tuber and root starches I. Structure and physicochemical properties of innala (Solenostemon rotundifolius) starches grown in Sri Lanka. Food Research International, 38: 615-629.

Lakušić B, Lakušić D, Jančić R, Stevanović B. 2006. Morpho-anatomical differentiation of the Balkan populations of the species Teucrium falvum $\mathrm{L}$. (Lamiaceae). Flora, 201: 108-119.

Lisboa ACCD, Mello ICM, Nunes RS, Dos Santos MA, Antoniolli AR, Marçal RM, Cavalcanti SC. 2006. Antinoceptive effect of Hyptis pectinata leaves extracts. Fitoterapia, 77: 439-442.

Martyrosyan EV, Ryzhova NN, Kochieva EZ. 2007. Polymorphism of Chloroplast Microsatellite DNA Loci in Russian Potato Cultivars Russian. Journal of Genetics, 43(11): 1325-1327.

Nanéma KR, Traoré RE, Bationo/Kando P, Zongo J-D. 2009. Morphoagronomical characterization of Solenostemon rotundifolius (Poir J. K. Morton) (Lamiaceae) germplasm from Burkina Faso. Int. J. Biol. Chem. Sci., 3(5): 11001113.

NRI. 1987. Root Crops (2nd edn). Tropical Development and Research Institute.

Odalo JO, Omolo MO, Malebo H, Angira J, Njeru PM, Ndiege IO, Hassanali A. 2005. Reppellency of essential oils of some plants from the Kenyan coast against Anopheles gambiae. Acta Tropica, 95: 210-218.

Opoku-Agyeman MO, Bennett-Lartey SO, Vodouhe RS, Osei C, Quarcoo E, 
Boateng SK, Osekere EA. 2007. Morphological characterization of frafra potato (Solenostemon rotundifolius) germplasm from the savannah regions of Ghana. Plant Genetic Resources and Food Security in West and Central Africa Regional Conference, Ibadan, Nigeria, 26-30 April, 2004; 116-123.

Ouédraogo A, Sédego A, Zongo JD. 2007. Perceptions paysannes de la culture et des utilisations du «fabirama» (Solenostemon rotundifolius (Poir) J.K. Morton) dans le plateau central du Burkina Faso. Ann. Bot. Afr. Ouest, 4: 13-21.

Owuor BO, Kisangau DP. 2005. Kenyan medicinal plants used as antivenin: a comparison of plant usage. Journal of Ethnobiology and Ethnomedicine, 2:7.

Pastorino MJ, Marchelli P, Milleron M, Soliani C, Gallo LA. 2009. The effect of different glaciation patterns over the current genetic structure of the southern beech Nothofagus Antarctica, Genetica 136:79-88.

Petitpierre B, Pairon M, Broennimann O, Jacquemart AL, Guisan A, Besnard G. 2009. Plastid DNA variation in Prunus serotina var serotina (Rosaceae), a North American tree invading Europe. Eur. J. Forest Res., 128: 431-436.

Powell W, Morgante M, McDevitt R, Vendramin GG, Rafalski JA. 1995. Polymorphic simple sequence repeat regions in chloroplast genomes: Applications to the population genetics of pines. Proc. Natl. Acad. Sci. USA, 92: 7759-7763.

Prematilake DP. 2005. Inducing genetic variation of innala $(S$. rotundifolius) via in vitro callus culture. J. Natn. Science Foundation Sri Lanka, 33(2): 123-131.

Provan J, Biss PM, Mc Meel D, Mathews S. 2004. Universal primers for the amplification of chloroplast microsatellites in grasses (Poaceae). Molecular Ecology Notes, 4: 262-264.

Rachmayanti Y, Leinemann L, Gailing O, Finkeldey R. 2006. Extraction, amplification and characterization of wood DNA from Dipterocarpaceae. Plant Molecular Biology Reporter, 24: 45-55.

Raimondi JP, Peralta IE, Masuelli RW, Feingold S, Camadro EL. 2005.
Examination of the hybrid origin of the wild potato Solanum ruiz-lealii Brücher. Pl. Syst. Evol., 253: 33-51.

Ryding O. 1994. The importance of pericarp structure in the classification of labiates. Lamiales Newsletter, 3: 1-3.

Ryzhova NN, Kochieva EZ. 2004. Analysis of microsatellite loci of the chloroplast genome in the genus Capsicum (Pepper). Russian Journal of Genetics, 40(8): 892896.

Schoeninger MJ, Bunn HT, Murray SS, Marlett JA. 2000. Composition of tubers used by hadza foragers of Tanzania. Journal of Food Composition and Analysis, 13: 1-12.

Sukhotu T, Hosaka K. 2006. Origin and evolution of Andigena potatoes revealed by chloroplast and nuclear DNA markers. Genome, 49: 636-647.

Tanzubil PB, Alem A, Zakariah M. 2005. Agronomic performance and pests of frafra potato (Solenostemon rotundifolius) in the Sudan savannah of Ghana. Tropical Science, 45: 10-13.

Trusty JL, Olmstead RG, Bogler DJ, SantosGuerra A, Francisco-Ortega J. 2004. Using molecular data to test a biogeographic connection of the Macaronesian genus Bystropogon (Lamiaceae) to the new world: a case of conflicting phylogenies. Systematic Botany, 29(3): 702-715.

Uniyal SK, Singh KN, Jamwal P, Lal B. 2006. Traditional use of medicinal plants among the tribal communities of Chhota Bhangal, Western Himalaya. Journal of Ethnobiology and Ethnomedicine, 2: 14.

Wagstaff SJ, Olmstead RG. 1997. Phylogeny of Labiatae and Verbenaceae inferred from $r b c L$ sequences. Sytematic Botany, 22(1): 165-179.

Weising K, Gardner RC. 1999. A set of conserved PCR primers for the analysis of simple sequence repeat polymorphisms in chloroplast genomes of dicotyledoneous angiosperms. Genome, 42: 9-19.

Wills DM; Burke JM. 2006. Chloroplast DNA variation confirms a single origin of domesticated sunflower (Helianthus annuus L.). Journal of Heredity, 97(4): 403-4. 